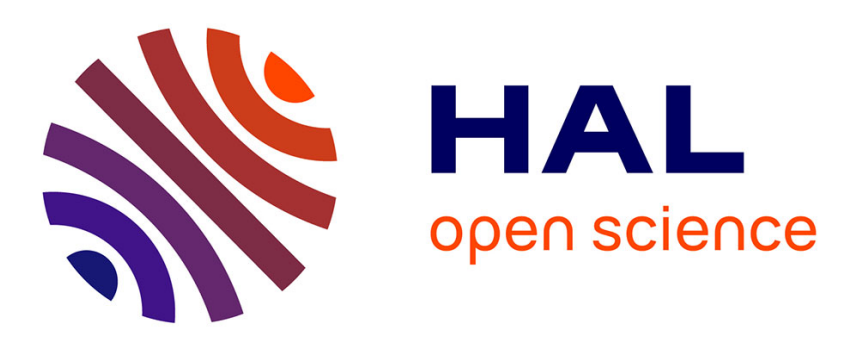

\title{
Optic Flow-Based Nonlinear Control and Sub-optimal Guidance for Lunar Landing
}

Guillaume Sabiron, Laurent Burlion, Thibaut Raharijaona, Franck Ruffier

\section{To cite this version:}

Guillaume Sabiron, Laurent Burlion, Thibaut Raharijaona, Franck Ruffier. Optic Flow-Based Nonlinear Control and Sub-optimal Guidance for Lunar Landing. IEEE International Conference on Robotics and Biomimetics, Dec 2014, Bali, Indonesia. hal-01079488

\section{HAL Id: hal-01079488 https://hal.science/hal-01079488}

Submitted on 2 Nov 2014

HAL is a multi-disciplinary open access archive for the deposit and dissemination of scientific research documents, whether they are published or not. The documents may come from teaching and research institutions in France or abroad, or from public or private research centers.
L'archive ouverte pluridisciplinaire HAL, est destinée au dépôt et à la diffusion de documents scientifiques de niveau recherche, publiés ou non, émanant des établissements d'enseignement et de recherche français ou étrangers, des laboratoires publics ou privés. 


\title{
Optic Flow-Based Nonlinear Control and Sub-optimal Guidance for Lunar Landing
}

\author{
Guillaume Sabiron $^{1,2}$, Laurent Burlion ${ }^{2}$, Thibaut Raharijaona ${ }^{1}$, and Franck Ruffier ${ }^{1}$
}

\begin{abstract}
A sub-optimal guidance and nonlinear control scheme based on Optic Flow (OF) cues ensuring soft lunar landing using two minimalistic bio-inspired visual motion sensors is presented here. Unlike most previous approaches, which rely on state estimation techniques and multiple sensor fusion methods, the guidance and control strategy presented here is based on the sole knowledge of a minimum sensor suite (including OF sensors and an IMU). Two different tasks are addressed in this paper: the first one focuses on the computation of an optimal trajectory and the associated control sequences, and the second one focuses on the design and theoretical stability analysis of the closed loop using only OF and IMU measurements as feedback information. Simulations performed on a lunar landing scenario confirm the excellent performances and the robustness to initial uncertainties of the present guidance and control strategy.
\end{abstract}

\section{INTRODUCTION}

During the last few decades, increasing attention has been paid to autonomous planetary landing, especially small lander applications requiring few resources for use in situations where mass, size and low-consumption embedded devices are of crucial importance. Applications of this kind always require a Guidance Navigation and Control (GNC) algorithm and finely tuned sensors which are able to bring the lander gently onto the ground. Minimalistic vision based systems equipped with lightweight bio-inspired sensors providing rich sensory feedback are particularly suitable for this purpose. Many authors have used vision based systems for various applications such as terrain relative navigation (see [1]), automatic landing, 3-D environment mapping and hazard avoidance. However, in most of these recent developments, a high computational cost is associated with the image processing algorithm extracting visual cues from the onboard cameras' output.

Bio-inspired devices have provided interesting solutions based on the Optic Flow (OF) cues which convey information about the relative velocity and the proximity of obstacles. The OF has been used in several studies to perform hazardous tasks such as taking off, terrain-following, and landing safely and efficiently by mimicking insects' behavior (see

\footnotetext{
* This research work is co-funded by CNRS Institutes (Life Science; Information Science; Engineering Science and Technology), the Aix-Marseille University, European Space Agency, ONERA the French Aerospace Lab and Astrium Satellites under ESA's Networking/Partnering Initiative program (NPI) for advanced technologies for space.

1 G. Sabiron, T. Raharijaona and F. Ruffier are with Aix-Marseille Université, CNRS, ISM UMR 7287, 13288 Marseille Cedex 09, France \{Thibaut.Raharijaona, Franck.Ruffier\}euniv-amu.fr.

${ }^{2}$ G. Sabiron, and L. Burlion are with the French Aerospace Lab (ONERA, Systems Control and Flight Dynamics -DCSD-), 31055 Toulouse, France \{Guillaume.Sabiron, Laurent.Burlion\}@onera.fr
}

[2], [3]), avoiding frontal obstacles (see [4]-[7]), tracking a moving target (see [8]) and hovering and landing on a moving platform (see [9]). We previously tested a miniature 2.8 g 6-pixel OF sensor implemented on a $80 \mathrm{~kg}$ helicopter by flying it outdoors over various fields, with promising results $[10]$.

OF based lunar landing has been addressed in several studies using either a nonlinear observer coupled to a Linear Quadratic (LQ) controller to track a constant OF reference in [11] or Proportional Integral Derivative (PID) type controllers to track a constant OF reference in [12], and more recently, using a Model Predictive Control approach in [13]. After presenting theoretical results on OF based optimal control in [14], previous authors adopted an OF reference signal based on the expansion OF (an index to the vertical velocity divided by the height) which was no longer constant, but decreased constantly or exponentially (see [15]).

In the present study, trajectory tracking was performed using a precomputed fuel-optimal trajectory assessed via nonlinear programming methods in order to avoid the unnecessary fuel expenditure liable to occur when following constant bio-inspired OF reference signals. In the control laws adopted, a rigorous Lyapunov approach was used to ensure the global stability and convergence of the closed loop including two nonlinear controllers based on translational and expansional OF measurements.

This paper is structured as follows. In section II, the dynamic model for the lander and the mathematical definition of the OF are presented. Section III describes the scenario studied and discusses the sub-optimal guidance scheme. Section IV presents the control strategy used for OF tracking purposes. Section V gives the results of the numerical simulations performed. Lastly, section VI contains some concluding comments and outlines our forthcoming projects.

\section{Lunar LANDER DyNAMIC Modeling AND OptiC FLOW EQUATIONS}

In this section, the dynamic model for the system presented in Fig. 2 and the mathematical background to OF studies are described. The autopilot presented here consists of an OF-based control system operating in the vertical plane $\left(\overrightarrow{e_{x}}, \overrightarrow{e_{z}}\right)$ (2-D position plus 1-D attitude), which controls the spacecraft's main thruster force and pitch angle. To stabilize the lander, it is necessary to cope with nonlinearities and the inherent instability of the system. Since the lunar atmosphere is very thin, no friction or wind forces are applied here to the 


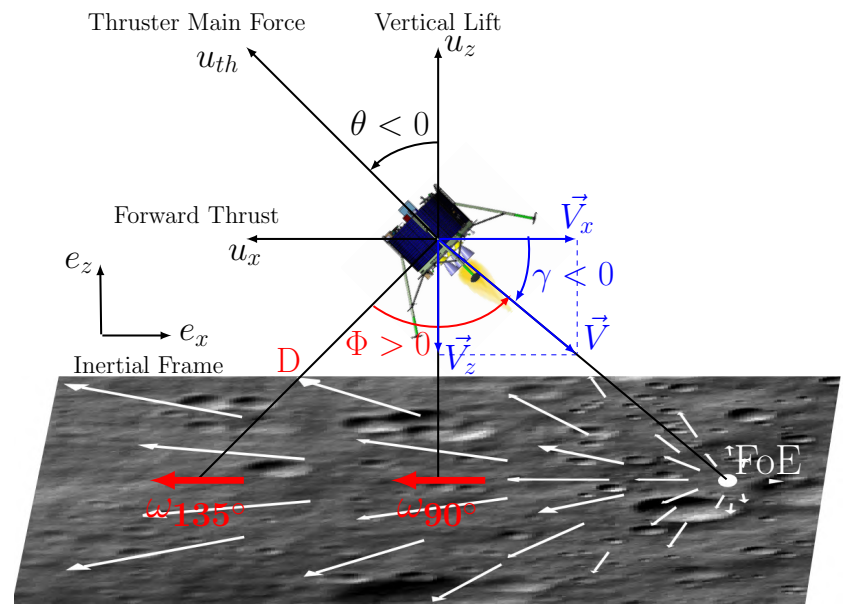

Fig. 1. Diagram of the lander, showing the inertial reference frame $\left(\overrightarrow{e_{x}}, \overrightarrow{e_{z}}\right)$, the velocity vector $\vec{V}$, the Focus of Expansion (FoE), and the mean thruster force $u_{t h}$ and its projections in the Local Vertical Local Horizontal (LVLH) reference frame. $\omega_{90}$ and $\omega_{135}$ are presented in red on the lunar ground. Adapted from [16].

lander. In line with previous studies, the lunar ground is taken to be flat (with an infinite radius of curvature) (see [17]). The lander's dynamic motion of the lander can be described in the time domain by the following dynamic system in the inertial frame $\left(\mathcal{I}\right.$ associated with the vector basis $\left.\left(\overrightarrow{e_{x}}, \overrightarrow{e_{z}}\right)\right)$ :

$$
\left\{\begin{array}{l}
\dot{V}_{x}(t)=\frac{\sin (\theta(t))}{m_{l d r}(t)} u_{t h}(t) \\
\dot{x}=V_{x} \\
\dot{V}_{z}(t)=\frac{\cos (\theta(t))}{m_{l d r}(t)} u_{t h}(t)-g_{\text {Moon }} \\
\dot{z}=V_{z} \\
\ddot{\theta}(t)=\frac{R}{I} u_{\theta}(t) \\
\dot{m}_{l d r}(t)=\frac{-u_{t h}(t)}{I_{s p_{t h}} . g_{\text {Earth }}}+\frac{-\left|u_{\theta}(t)\right|}{I_{s p_{\theta}} . g_{\text {Earth }}}
\end{array}\right.
$$

where $0 \leq u_{t h} \leq 3820 N$ corresponds to the control force applied to the lander and $-44 \leq u_{\theta} \leq 44 N$ is the control input signal driving the spacecraft's pitch. $V_{x, z}$ are the lander's velocities in the lunar inertial reference frame, $m_{l d r}$ stands for the lander's mass, $\theta$ is the pitch angle, $t$ denotes the time, and $g_{M o o n}$ denotes the lunar acceleration due to the gravity $\left(g_{\text {Moon }}=1.63 \mathrm{~m} / \mathrm{s}^{2}, g_{\text {Moon }}\right.$ is taken to be constant due to the low initial altitude). $I$ is the lander's moment of inertia, and $R$ is the eccentricity of the attitude thrusters from the center of mass. $I_{s p}$ is the specific impulse: $I_{s p_{t h}}=325 \mathrm{~s}$ in the case of the braking thrusters, $I_{s p_{\theta}}=287 \mathrm{~s}$ in that of the attitude thrusters and $g_{\text {Earth }}=9.81 \mathrm{~m} / \mathrm{s}^{2}$ is the Earth's gravity. Numerical values are taken from ESA/ASTRIUM studies or in accordance with literature. In the vertical plane, the OF $\omega(\Phi)$ was defined by [18] as follows:

$$
\omega(\Phi)=\frac{V}{D} \sin (\Phi)-\dot{\theta}
$$

where the term $\frac{V}{D} \sin (\Phi)$, which is called the translational $\mathrm{OF}$, depends on the linear velocity $V$ expressed in the inertial frame, the distance from the ground $D$ in the gaze direction and the elevation angle $\Phi$ (i.e. the angle between the gaze direction and the heading direction). In order to use the useful properties of the translational OF, the angular velocity $\dot{\theta}$ corresponding to the rotational $\mathrm{OF}$ is subtracted from the measured OF $\omega_{\text {meas }}$, using IMU measurements: this operation is known as the derotation process (see [19]). For the sake of clarity, the two specific local translational OFs used in this study will be written as follows:

- $\omega_{90^{\circ}}$ stands for the downward translational OF, i.e. in the nadir direction $\left(90^{\circ}\right.$ between the gaze direction and the local horizontal) after the derotation, and

- $\omega_{135^{\circ}}$ stands for the translational OF oriented at an angle of $135^{\circ}$ with respect to the local horizontal after the derotation.

In this study, the sensors available were an IMU and two OF sensors oriented at angles of $90^{\circ}$ and $135^{\circ}$ with respect to the local horizontal in a fixed position whatever the lander's attitude thanks to a gimbal system.

From (2), under the assumption that the ground is practically flat (i.e. $D=h / \cos \left(\frac{\pi}{2}-\Phi+\gamma\right)$, where $\gamma$ denotes the flight path angle (the orientation of the velocity vector with respect to the local horizontal), $h$ is the ground height, and $\Phi-\gamma$ is the angle between the gaze direction and the local horizontal:

$$
\begin{gathered}
\omega_{90^{\circ}}=\frac{V_{x}}{h} \\
\omega_{135^{\circ}}=\frac{V}{2 h}(\cos (\gamma)-\sin (\gamma))=\frac{\omega_{90^{\circ}}}{2}(1-\tan (\gamma))
\end{gathered}
$$

where $\tan (\gamma)=\frac{V_{z}}{V_{x}}$. The highly informative OF values, that is to say, those of the ventral OF $\omega_{x}$ and the expansion OF $\omega_{z}$ used in the newly developed regulators are then expressed directly in terms of $\omega_{90^{\circ}}$ and $\omega_{135^{\circ}}$ :

$$
\begin{gathered}
\omega_{x}=\frac{V_{x}}{h}=\omega_{90^{\circ}} \\
\omega_{z}=\frac{V_{z}}{h}=\omega_{90^{\circ}}-2 \omega_{135^{\circ}}
\end{gathered}
$$

\section{Sub-optimal Guidance Strategy}

Here it is proposed to study autonomous landing during the approach phase extending from the High Gate (HG) $-1800 \mathrm{~m}$ AGL- to the Low Gate (LG) -10m AGL. The mass optimization problem was defined here along with the constraints involved, and its solution was computed in terms of the trajectory and the OF profiles. In order to meet the low computational requirements, the optimal problem was solved offline only once: the OF and pitch profiles were determined and implemented in the form of constant vectors in the lander. Therefore, the guidance strategy is said to be sub-optimal since the offline computed optimal trajectory correspond to the nominal initial conditions which may not be met at the HG.

First of all, the optimal control sequence $u^{*}=$ $\left(u_{t h}^{*}, u_{\theta}^{*}\right)$ was computed, taking $u_{t h}^{*}$ to denote the braking thrust and $u_{\theta}^{*}$ to denote the pitch torque (the upper script 


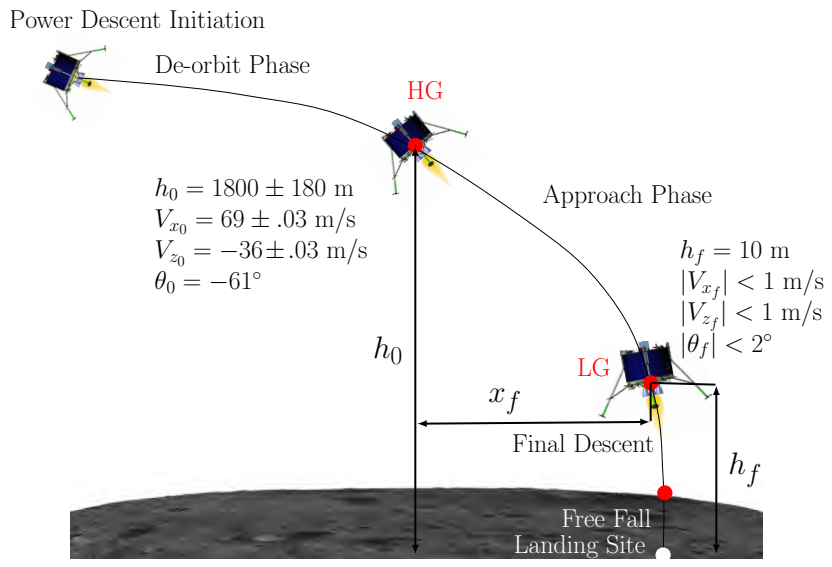

Fig. 2. The lander's objectives are to reach LG (10 m high) with both vertical and horizontal velocities of less than $1 \mathrm{~m} / \mathrm{s}$ in absolute values and a pitch angle in the $\pm 2^{\circ}$ range. Adapted from [16].

* indicates the optimality in terms of the mass, i.e., the fuel consumption). In this paper, optimality refers to the outputs of the optimization problem $\left(u_{t h}^{*}, u_{\theta}^{*}\right)$ and the associated reference trajectory $\left(\dot{V}_{x}^{*}, \dot{V}_{z}^{*}, V_{x}^{*}, V_{z}^{*}, h^{*}, \theta^{*}\right)$. Looking for the least fuel-consuming trajectory is equivalent to finding the control sequence $u^{*}$ that minimizes the use of the control signal (see (1f)). The optimization problem can then be expressed as follows:

Solve

$$
\min _{u_{t h}(t), u_{\theta}(t)} \int_{t_{0}}^{t_{f}}\left(u_{t h}(t)+\left|u_{\theta}(t)\right|\right) \mathrm{d} t
$$

\section{Subject to}

Equations (1a)-(1f)

$$
\begin{aligned}
& \begin{cases}V_{z}\left(t_{0}\right)=-36 \mathrm{~m} / \mathrm{s}, & \left|V_{z_{f}}\right|<1 \mathrm{~m} / \mathrm{s} \\
V_{x}\left(t_{0}\right)=69 \mathrm{~m} / \mathrm{s}, & \left|V_{x_{f}}\right|<1 \mathrm{~m} / \mathrm{s} \\
h\left(t_{0}\right)=1800 \mathrm{~m}, & h_{f}=10 \mathrm{~m} \\
\theta\left(t_{0}\right)=-61^{\circ}, & \left|\theta_{f}\right|<2^{\circ}\end{cases} \\
& \left\{\begin{array}{l}
0<u_{t h}<3438 \mathrm{~N} \\
-44<u_{\text {pitch }}<44 \mathrm{~N} \quad \forall t \in\left[t_{0}, t_{f}\right] \\
\left(-V_{z}, V_{x}, h, x\right)>0 \\
|\dot{\theta}|<1.5^{\circ} / \mathrm{s}
\end{array}\right.
\end{aligned}
$$

This offline sub-optimal guidance strategy was implemented using Matlab optimization software on the nonlinear system under constraints to bring the system from HG to LG. To solve this continuous time optimization problem, many freely available Matlab toolboxes based on various methods can be used. The solution provided by ICLOCS (Imperial College London Optimal Control Software, [20]) based on the IPOPT solver suited our needs for the numerical implementation of a nonlinear optimization problem in the case of the continuous system subjected to boundary and state constraints using the interior point method. The simulation of the open loop under optimal control was therefore run on the nonlinear system to assess the optimal OF and pitch profiles $\left(\omega_{x}^{*}, \omega_{z}^{*}, \theta^{*}\right)$.
Equation (1a)-(1f) describes the dynamic lander, (8) gives the initial and final conditions and (9) gives the actuator and system constraints imposed along the trajectory. For safety reasons, a $10 \%$ margin was added to the thrusters' physical saturation in order to give the lander greater maneuverability around the predefined trajectory at any point. It is worth noting that a terminal constraint could easily be added if required to the downrange $x$ to make pinpoint landing possible, but this might greatly increase the fuel consumption. Since the case may arise where $\dot{\theta}=-\omega_{R}>\omega_{T}$ and thus $\omega_{\text {measured }}<0$, we had to use a bi-directional version of the 6-pixel VMS adapted for use in the following measurement range: $\omega_{\text {measured }} \epsilon\left[-20^{\circ} / \mathrm{s} ;-0,1^{\circ} / \mathrm{s}\right] \cup\left[0,1^{\circ} / \mathrm{s} ; 20^{\circ} / \mathrm{s}\right]$. The fuel expenditure decreases the lander's mass by $\Delta m$, which is defined as the difference between the initial and final mass of the lander $\Delta m=m_{l d r_{0}}-m_{l d r}\left(t_{f}\right)$ where $m_{l d r_{0}}=762$ $\mathrm{kg}$ and

$m_{l d r}\left(t_{f}\right)=m_{l d r}\left(t_{0}\right)-\frac{1}{g_{E a r t h}} \int_{t_{0}}^{t_{f}}\left(\frac{u_{t h}(\epsilon)}{I_{s p_{t h}}}+\frac{\left|u_{\theta}(\epsilon)\right|}{I_{s p_{\theta}}}\right) d \epsilon$

In order to make sure that the sum $\omega_{\text {grd }-t r h}=\omega_{T}+\omega_{R}$ does not cancel itself out (i.e. $\left.\omega_{T}=-\omega_{R}\right)$, the pitch rate $\left(\omega_{R}=\dot{\theta}\right)$ was constrained as follows: $|\dot{\theta}|=\left|\omega_{R}\right|<1.5^{\circ} / \mathrm{s}$. Under all these conditions, the optimal control sequences $\left(u_{t h}^{*}, u_{\theta}^{*}\right)$ were processed: the optimal solution was obtained with $t_{f}=$ $51.46 \mathrm{~s}$ and a mass change of $\Delta m<33.6 \mathrm{~kg}$ (amounting to $4.4 \%$ of the initial mass). The trajectory modeled under these constraints can be said to be optimal in the case of a more highly constrained problem. Additional constraints were imposed on $\dot{\theta}$ and the $10 \%$ margin on the thrust to account for the sensors' and actuators' operating ranges, resulting in a more highly constrained problem than the system can actually deal with. In any case, both of these constraints (the saturated pitch rate and the $10 \%$ margin added to the thrust) resulted in very similar fuel expenditure predictions to that obtained without these additional constraints (amounting to a difference of only $0.21 \%$ ).

\section{LYAPUNOV-BASED NONLINEAR CONTROL DESIGN}

In this section, a control design ensuring soft lunar landing based on the knowledge of the OF and IMU measurements is presented. The control problem to be solved here focuses on the tracking of translational and expansional OF reference signals. In particular, two control signals are computed, one for the horizontal thrust $u_{x}$ and one for the vertical thrust $u_{z}$. Both $u_{x}$ and $u_{z}$ are then fused into a jointly delivered control signal $u_{t h}=\sqrt{u_{x}^{2}+u_{z}^{2}}$.

\section{A. Height boundedness}

Here we look for a time varying bound on the height $h$ From:

$$
\omega_{z}=\frac{V_{z}}{h}=\frac{d}{d t} \ln (h)
$$

we have

$$
\int_{t_{0}}^{t} \omega_{z}(s) \mathrm{d} s=\ln \left(\frac{h(t)}{h\left(t_{0}\right)}\right)
$$




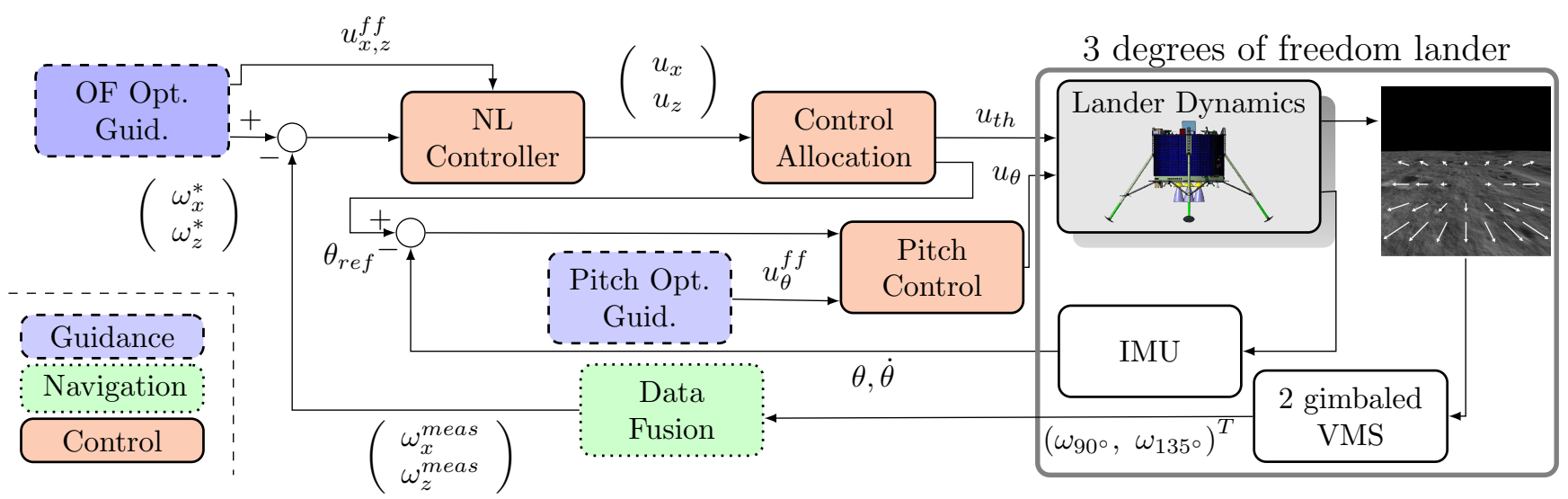

Fig. 3. Sketch of the full GNC solution. The dynamic model for the lander with $2 \times 6$-pixels VMS feeding the data fusion block along with an IMU. The data fusion block estimates high interest OF values, which are conveyed to the nonlinear controller. The control allocation block transforms the control signal into a braking force defining the magnitude of the thrust vector and a reference pitch angle. The inner attitude control loop delivers the torque control signal $u_{\theta}$ assessed via the linear output feedback controller and a sub-optimal guidance strategy defining the feedfoward term (corresponding to $u_{x}^{f f}=\dot{V}_{x}^{*}$ and $u_{z}^{f f}=\dot{V}_{z}^{*}$ in the control law equations). Adapted from [16].

which gives

$$
h(t)=h\left(t_{0}\right) \mathrm{e}^{\int_{t_{0}}^{t} \omega_{z}(s) \mathrm{d} s}
$$

where $\omega_{z}(t)<0$ and $h(t)>0$. Since it can be deduced from the initial conditions that $h\left(t_{0}\right) \in[1620,1980]$, from (13) it is thus possible to compute time-varying bounds on the height such that $\forall t \geq t_{0} h(t) \in\left[h_{\min }(t), h_{\max }(t)\right]$ where:

$$
\left\{\begin{array}{l}
h_{\text {min }}(t)=1620 \mathrm{e}^{\int_{t_{0}}^{t} \omega_{z}(s) \mathrm{d} s} \\
h_{\text {max }}(t)=1980 \mathrm{e}^{\int_{t_{0}}^{t} \omega_{z}(s) \mathrm{d} s}
\end{array}\right.
$$

which means that at each time step, an upper and a lower bound on $h(t)$ depending on the uncertainty at $\mathrm{HG}$ are known.

Remark If the measurement $\omega_{z}^{\text {meas }}(s)$ is corrupted with noise i.e. $\omega_{z}^{\text {meas }}(s)-d \leq \omega_{z}(s)<\omega_{z}^{\text {meas }}(s)+d<0$ where $d \geq 0$ then

$$
\left\{\begin{array}{l}
h_{\text {min }}(t)=1620 e^{\int_{t_{0}}^{t} \omega_{z}^{\text {meas }}(s)-d d s} \\
h_{\text {max }}(t)=1980 e^{\int_{t_{0}}^{t} \omega_{z}^{\text {meas }}(s)+d d s}
\end{array}\right.
$$

\section{B. Z dynamics}

The nonlinear control design is achieved using the following Lyapunov function candidate $\mathcal{L}_{1}$ so that (see [21]):

$$
\mathcal{L}_{1}=\frac{1}{2} S_{z}^{2}
$$

where $S_{z}$ is defined such as $S_{z}=\omega_{z}-\omega_{z}^{*}$.

Its corresponding time derivative might therefore be expressed as follows:

$$
\begin{gathered}
\dot{\mathcal{L}}_{1}=S_{z}\left[\frac{\dot{V}_{z}}{h}-\frac{\dot{V}_{z}^{*}}{h^{*}}-\omega_{z}^{2}+\omega_{z}^{*^{2}}\right] \\
\dot{\mathcal{L}}_{1}=S_{z}\left[\frac{\dot{V}_{z}-\dot{V}_{z}^{*}}{h}+\dot{V}_{z}^{*}\left(\frac{1}{h}-\frac{1}{h^{*}}\right)-S_{z}^{2}-2 \omega_{z}^{*} S_{z}\right]
\end{gathered}
$$

Two possible cases then arise, depending on the sign of $S_{z}$. A sign study of $S_{z}$ gives:

- When $S_{z}>0$

$$
\dot{\mathcal{L}}_{1}<S_{z}\left[\frac{\dot{V}_{z}-\dot{V}_{z}^{*}}{h}+\dot{V}_{z}^{*}\left(\frac{1}{h}-\frac{1}{h^{*}}\right)-2 \omega_{z}^{*} S_{z}\right]
$$

where $h>0$ in the reference scenario under consideration, using bounds on $h(t)$ such that:

$\frac{1}{h_{\max }(t)}-\frac{1}{h^{*}(t)} \leq\left(\frac{1}{h(t)}-\frac{1}{h^{*}(t)}\right) \leq \frac{1}{h_{\min }(t)}-\frac{1}{h^{*}(t)}$

This gives

$$
\dot{\mathcal{L}}_{1} \leq S_{z}\left(\frac{\dot{V}_{z}-\dot{V}_{z}^{*}}{h}\right)+\left|S_{z}\right|\left|\dot{V}_{z}^{*}\right| h_{\text {bound }}+\left|2 \omega_{z}^{*}\right| S_{z}^{2}
$$

where

$$
h_{\text {bound }}=\max \left(\left|\frac{1}{h_{\max }(t)}-\frac{1}{h^{*}}\right|,\left|\frac{1}{h_{\min }(t)}-\frac{1}{h^{*}}\right|\right)
$$

We now need to find a control signal satisfying $\dot{\mathcal{L}}_{1}<0$. The virtual control signal $u_{z}$ features in the dynamic model for the lander in the form of $\dot{V}_{z}=\frac{u_{z}}{m}-g_{\text {Moon }}$, where $u_{z}=\cos (\theta) u_{t h}$. We take:

$$
u_{z}(t)=m\left(\dot{V}_{z}^{*}-k_{a}(t) S_{z}-k_{b}(t) \operatorname{sgn}\left(S_{z}\right)+g_{M o o n}\right)
$$

where $\operatorname{sgn}(X)=\left\{\begin{array}{ll}1 & X \geq 0 \\ -1 & X<0\end{array}\right.$.

We obtain

$$
\dot{\mathcal{L}}_{1} \leq S_{z}^{2}\left(\frac{-k_{a}(t)}{h}+\left|2 \omega_{z}^{*}\right|\right)+\left|S_{z}\right|\left(\frac{-k_{b}(t)}{h}+\left|\dot{V}_{z}^{*}\right| h_{\text {bound }}\right)
$$

Lastly, we take the gains $k_{a}(t)$ and $k_{b}(t) \forall t \geq 0$ :

$$
\begin{gathered}
k_{a}(t)>h_{\max }(t)\left|2 \omega_{z}^{*}(t)\right| \\
k_{b}(t)>h_{\max }(t)\left|\dot{V}_{z}^{*}(t)\right| h_{\text {bound }}
\end{gathered}
$$


so that $\dot{\mathcal{L}}_{1}<0$.

- When $S_{z}<0$

From equation (17), one can obtain

$$
\dot{\mathcal{L}}_{1} \leq S_{z}\left(\frac{\dot{V}_{z}-\dot{V}_{z}^{*}}{h}\right)+\left|S_{z}\right|\left|\dot{V}_{z}^{*}\right| h_{\text {bound }}+\left|2 \omega_{z}^{*}\right| S_{z}^{2}-S_{z}^{3}
$$

We now need to find a control signal satisfying $\dot{\mathcal{L}}_{1}<0$ We take:

$u_{z}(t)=m\left(\dot{V}_{z}^{*}-k_{a}(t) S_{z}-k_{b}(t) \operatorname{sgn}\left(S_{z}\right)-k_{c}(t) S_{z}^{2}+g_{M o o n}\right.$

Hence

$$
\begin{aligned}
\dot{\mathcal{L}}_{1} \leq & S_{z}\left(\frac{-k_{a}(t) S_{z}-k_{b}(t) \operatorname{sgn}\left(S_{z}\right)-k_{c}(t) S_{z}^{2}}{h}\right) \\
+ & S_{z}|| \dot{V}_{z}^{*}\left|h_{\text {bound }}+\right| 2 \omega_{z}^{*} \mid S_{z}^{2}-S_{z}^{3} \\
\dot{\mathcal{L}}_{1} \leq & S_{z}^{2}\left(\frac{-k_{a}(t)}{h}+\left|2 \omega_{z}^{*}\right|\right) \\
& +\left|S_{z}\right|\left(\frac{-k_{b}(t)}{h}+\left|\dot{V}_{z}^{*}\right| h_{\text {bound }}\right) \\
& -S_{z}^{3}\left(1+\frac{k_{c}(t)}{h}\right)
\end{aligned}
$$

where we choose the gain $k_{c}(t) \forall t \geq 0$ so that:

$$
k_{c}(t)<-h_{\max }(t)
$$

and $k_{a}(t), k_{b}(t)$ such as (29-29) are ensured.

Therefore $\dot{\mathcal{L}}_{1}<0$, which means that $\mathcal{L}_{1}$ tends asymptotically toward 0 (since $\mathcal{L}_{1}>0$ ), and lastly, (15) ensures that $\omega_{z} \rightarrow$ $\omega_{z}^{*}$ asymptotically.

Let us now combine all the expressions for the control signal, with (22-27) to obtain the unified control signal equation:

$$
\begin{aligned}
u_{z}(t)= & m\left(\dot{V}_{z}^{*}-k_{a}(t) S_{z}-k_{b}(t) \operatorname{sgn}\left(S_{z}\right)\right. \\
& \left.-\left(\frac{1-\operatorname{sgn}\left(S_{z}\right)}{2}\right) k_{c}(t) S_{z}^{2}+g_{M o o n}\right)
\end{aligned}
$$

\section{X dynamics}

A similar Lyapunov function based approach is used on the X dynamics:

Let us define $S_{x}$ as $S_{x}=\omega_{x}-\omega_{x}^{*}$ :

$$
\begin{gathered}
\mathcal{L}_{2}=\frac{1}{2} S_{x}^{2} \\
\dot{\mathcal{L}}_{2}=S_{x}\left[\frac{\dot{V}_{x}}{h}-\frac{\dot{V}_{x}^{*}}{h^{*}}-\omega_{x} \omega_{z}+\omega_{x}^{*} \omega_{z}^{*}\right]
\end{gathered}
$$

One can say that:

$$
\dot{\mathcal{L}}_{2}<S_{x}\left[\frac{\dot{V}_{x}-\dot{V}_{x}^{*}}{h}+\dot{V}_{x}^{*}\left(\frac{1}{h}-\frac{1}{h^{*}}\right)+\omega_{x} \omega_{z}+\omega_{x}^{*} \omega_{z}^{*}\right]
$$

with $h>0$ in the reference scenario adopted:

$$
\frac{1}{h_{\max }(t)}-\frac{1}{h^{*}} \leq\left(\frac{1}{h}-\frac{1}{h^{*}}\right) \leq \frac{1}{h_{\min }(t)}-\frac{1}{h^{*}}
$$

This gives:

$$
\dot{\mathcal{L}}_{2} \leq S_{x}\left(\frac{\dot{V}_{x}-\dot{V}_{x}^{*}}{h}\right)+\left[\left|\dot{V}_{x}^{*}\right| h_{\text {bound }}+\left|\omega_{x} \omega_{z}+\omega_{x}^{*} \omega_{z}^{*}\right|\right]\left|S_{x}\right|
$$

where $h_{\text {bound }}$ as defined in (21).

We need to find a control signal that ensure $\dot{\mathcal{L}}_{2}<0$. The virtual control signal $u_{x}$ features in the dynamic model for the lander in the form of $\dot{V}_{x}=\frac{u_{x}}{m}$, where $u_{x}=\sin (\theta) u_{t h}$. We choose:

$$
u_{x}(t)=m\left(\dot{V}_{x}^{*}-k_{a}(t) \operatorname{sgn}\left(S_{x}\right)-k_{b} S_{x}\right)
$$

where $\forall t \geq 0$

$$
k_{a}(t)>h_{\max }(t)\left[\left|\dot{V}_{x}^{*}\right| h_{\text {bound }}+\left|\omega_{x} \omega_{z}+\omega_{x}^{*} \omega_{z}^{*}\right|\right]
$$

thus with $\dot{\mathcal{L}}_{2}<-k_{b} S_{x}$, we choose a relatively small $k_{b}>0$ to prevent any chattering of $S$ at values around zero.

Finally, $\dot{\mathcal{L}}_{2}<0$, which means that $\mathcal{L}_{2}$ tends asymptotically toward 0 (since $\mathcal{L}_{2}>0$ ), and lastly, (32) ensures that $\omega_{x} \rightarrow$ $\omega_{x}^{*}$ asymptotically.

\section{Pitch control law}

To control the attitude, a proportional derivative controller drives the spacecraft's pitch (via the inner loop), which gives faster dynamics in the inner loop than on an outer loop:

$$
u_{\theta}(t)=u_{\theta}^{f f}(t)+K_{p} \varepsilon_{\theta}(t)+K_{d} \frac{d}{d t} \varepsilon_{\theta}(t)
$$

where $u_{\theta}^{f f}(t)$ corresponds to the optimal control sequence $u_{\theta}^{*}(t)$ computed with the mass-optimal trajectory and $\varepsilon_{\theta}(t)=$ $\theta_{\text {meas }}(t)-\theta_{\text {ref }}(t)$. The reference signal $\theta_{\text {ref }}$ is based on the two virtual control signals $u_{x}$ and $u_{z}$, so that:

$$
\theta_{\text {ref }}=\arctan \left(\frac{u_{x}}{u_{z}+\varepsilon}\right)
$$

where $\varepsilon$ is taken to be very small to avoid having to divide by zero.

\section{Simulation Results}

Once the optimal trajectory has been defined, the OF and pitch profiles $\left(\omega_{x}^{*}, \omega_{z}^{*}, \theta^{*}\right)$ as well as the optimal feedforward control signals $\dot{V}_{z}^{*}$ and $\dot{V}_{z}^{*}$ (see $(27,37)$ ) are available for implementation along with the control laws defined in (27), (37) and (39). Simulations were run on a Matlab/Simulink simulator taking the lander's dynamics, actuator dynamics (which were taken to be first order systems) and the saturation into account. Random noise was also added to the OF sensor model. In order to assess the robustness of the model to initial uncertainties, an initial height condition $\left(h\left(t_{0}\right)\right)$ was taken to be in the $1800 \pm \delta_{h}$ range, where $\delta_{h}=180 \mathrm{~m}$. The result of simulations in which $h\left(t_{0}\right)$ increased by $20 \mathrm{~m}$ after each run are presented in Fig. 4. As can be seen from this figure, which presents the trajectory in the 2-D plane and the final velocities, pitch angle and fuel consumption, our new G\&C almost meets the tight specifications imposed. The final vertical velocity is slightly higher than the objective. The final pitch angle was in the $\pm 2^{\circ}$ range, the horizontal velocity was below $1 \mathrm{~m} / \mathrm{s}$, whereas the final vertical velocity was only 0.68 $\mathrm{m} / \mathrm{s}$ above the objective (corresponding to a decrease in the speed of $100 \frac{V_{z_{f}}-V_{z}\left(t_{0}\right)}{V_{z}^{*}\left(t_{f}\right)-V_{z}\left(t_{0}\right)}=98 \%$ of the tight requirements) 

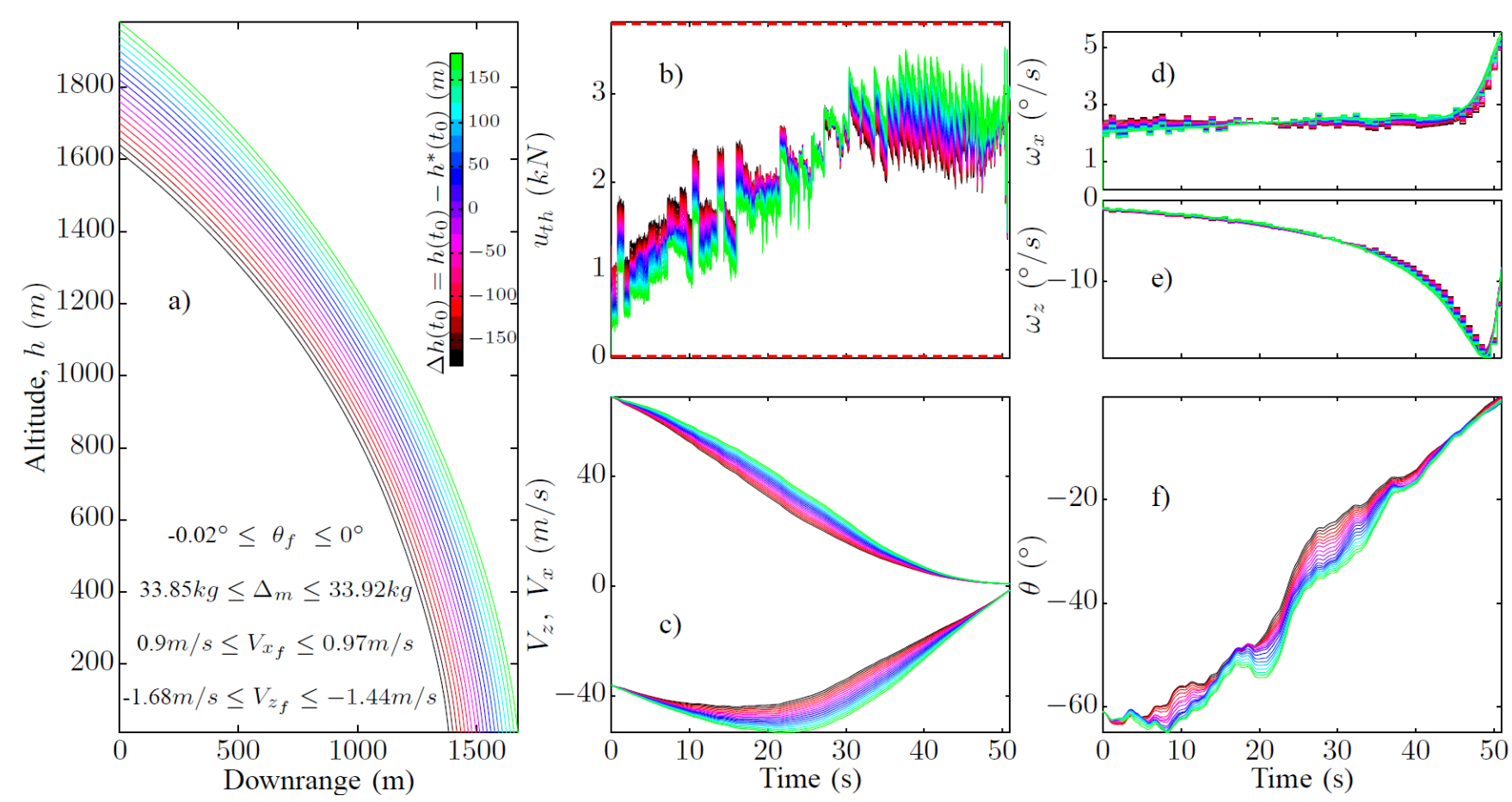

Fig. 4. Closed loop behavior from HG to LG in simulations with $h\left(t_{0}\right) \in\left[h_{\min }\left(t_{0}\right), h_{\max }\left(t_{0}\right)\right]$. a) Height $h$ versus downrange $x$. b) Control sequence $u_{t h}=\sqrt{u_{x}^{2}+u_{z}^{2}}$. Saturation of the control signal $u_{t h}$ is defined in such a way that $0 \mathrm{~N} \leq u_{t h} \leq 3820 \mathrm{~N}$. c) Velocities $V_{x}$, $V_{z}$. d) Measured OF and optimal reference OF profile (black dashed lines). e) Pitch trajectories corresponding to various initial heights.

in the worst simulated case. The fuel consumption was $\Delta m \leq 34.22 \mathrm{~kg}$, although we observed that $\Delta m^{*}=33.6 \mathrm{~kg}$, which means that even when the initial height was far above the pre-computed optimal trajectory, the fuel consumption approached the optimal value very closely (it was only $1.2 \%$ higher) although the final constraints were almost met. It is worth noting that the control signal $u_{t h}(t)$ presented in Fig. 4.b never reached the upper or lower saturation levels depicted in dashed red lines. The evolution of the velocities, which tended toward $1 \mathrm{~m} / \mathrm{s}$ in absolute values, is presented in Fig. 4.c, whereas Fig. 4.d-e shows the evolution of the optic flow measured superimposed on the optimal reference signals. Noise was modeled based on previous results obtained on the real sensor, which showed the occurrence of a refresh rate of approximately $7 \mathrm{~Hz}$ and a standard deviation of the error of $0.4^{\circ} / \mathrm{s}$. Lastly, Fig. 4.f presents the pitch evolution starting at $\theta\left(t_{0}\right)=-61^{\circ}$ and moving toward $-2^{\circ} \geq \theta_{f} \geq 2^{\circ}$ in the case of all the initial heights. In conclusion, the G\&C strategy presented in this study can be said to be suitable for handling the approach phase during lunar landing, even in the presence of large initial uncertainties as far as the height is concerned.

\section{CONCLUSION}

This paper presents a nonlinear soft lunar landing controller, in which optical flow measurements are used along with the IMU data. The originality of our approach lies in the fact that neither the linear velocity nor the distance from the target need to be determined. The present approach involves an image based visual control algorithm which requires only $2 \times 6$ pixels and inertial data for performing the derotation of the flow. A rigorous analysis of the stability of the closed-loop systems presented here was conducted, which resulted in the design of sliding mode type control laws regulating the translational and expansional OF. Via nonlinear programming procedures, the optimal reference trajectory in terms of the fuel consumption was computed offline and used in the closed loop as feedforward terms for providing the OF and pitch control loops with reference signals. The guidance algorithm proposed here is designated as sub-optimal in terms of fuel expenditure since it provides the system with an optimal trajectory from the HG to the LG computed via nonlinear programming. The actual landing strategy is therefore sub-optimal since the optimal trajectory is computed offline only once. In view of the simulation results we can conclude that the strategy is close to the optimal behavior. Simulations with various initial conditions gave a clear picture of the performances of the present algorithm. The experimental results obtained confirmed that the G\&C strategy developed here almost fulfilled the requirements in terms of the spacecraft's final position, velocity and fuel consumption. It is now proposed to conduct further research on the following lines. First, in order to do away with the use of bulky IMUs and gimbal systems, an observer based solely on the OF could be used to accurately estimate $\omega_{x}$, $\omega_{z}$ and $\theta$. An initial study on an observer of this kind was recently presented in [22]. Secondly, although the final pitch angle estimates meet the objectives, an improvement could be made by designing a nonlinear control law regulating the pitch dynamics in order to avoid having to differentiate the 
pitch error, which depends on the control signals.

\section{ACKNOWLEDGMENT}

We are most grateful to S. Viollet, G. Jonniaux, E. Kervendal and E. Bornschlegl for their fruitful suggestions and comments during this study. We thank J. Blanc for improving the English manuscript. This research work was co-funded by CNRS Institutes (Life Science; Information Science; Engineering Science and Technology), the Aix-Marseille University, European Space Agency, the French Aerospace Lab (ONERA), and the French Aerospace Lab and Airbus Defense and Space under ESA's Networking/Partnering Initiative program (NPI) for advanced technologies for space.

\section{REFERENCES}

[1] A. E. Johnson and J. F. Montgomery, "Overview of terrain relative navigation approaches for precise lunar landing," in Aerospace Conference, 2008 IEEE. IEEE, 2008, pp. 1-10.

[2] F. Ruffier and N. Franceschini, "Optic flow regulation: the key to aircraft automatic guidance," Robotics and Autonomous Systems, vol. 50, pp. 177-194, 2005.

[3] B. Hérissé, T. Hamel, R. Mahony, and F. Russotto, "A terrainfollowing control approach for a VTOL Unmanned Aerial Vehicle using average optical flow," Autonomous Robots, vol. 29, no. 3-4, pp. 381-399, 2010. [Online]. Available: http://dx.doi.org/10.1007/s10514010-9208-X

[4] G. Barrows and C. Neely, "Mixed-mode VLSI optic flow sensors for in-flight control of a Micro Air Vehicle," in SPIE : Critical technologies for the future of computing, vol. 4109, San Diego, CA, USA, Aug. 2000, pp. 52-63.

[5] S. Griffiths, J. Saunders, A. Curtis, B. Barber, T. McLain, and R. Beard, "Maximizing miniature aerial vehicles," IEEE Robotics \& Automation Magazine, vol. 13, pp. 34-43, 2006.

[6] F. Ruffier and N. Franceschini, "Aerial robot piloted in steep relief by optic flow sensors," in IEEE/RSJ International Conference on Intelligent Robots and Systems (IROS). IEEE, 2008, pp. 1266-1273.

[7] A. Beyeler, J. Zufferey, and D. Floreano, "OptiPilot: control of takeoff and landing using optic flow," in European Micro Aerial Vehicle Conference (EMAV), vol. 27, Delft, Nederlands, Sept. 2009.

[8] F. Kendoul, K. Nonami, I. Fantoni, and R. Lozano, "An adaptive vision-based autopilot for mini flying machines guidance, navigation and control," Autonomous Robots, vol. 27, pp. 165-188, 2009.
[9] B. Hérissé, T. Hamel, R. Mahony, and F.-X. Russotto, "Landing VTOL Unmanned Aerial Vehicle on a Moving Platform Using Optical Flow," IEEE Transactions on Robotics, vol. 28, no. 1, pp. 77 -89, Feb. 2012.

[10] G. Sabiron, P. Chavent, T. Raharijaona, P. Fabiani, and F. Ruffier, "Low-speed optic-flow sensor onboard an unmanned helicopter flying outside over fields," in IEEE International Conference on Robotics and Automation (ICRA), 2013.

[11] F. Valette, F. Ruffier, S. Viollet, and T. Seidl, "Biomimetic optic flow sensing applied to a lunar landing scenario," in International Conference on Robotics and Automation (ICRA), 2010, pp. 2253 2260.

[12] V. Medici, G. Orchard, S. Ammann, G. Indiveri, and S. Fry, "Neuromorphic computation of optic flow data Bio-inspired landing using biomorphic vision sensors," ESA, Tech. Rep., 2010.

[13] D. Izzo and G. de Croon, "Nonlinear model predictive control applied to vision-based spacecraft landing," in Proceedings of the EuroGNC 2013, 2nd CEAS Specialist Conference on Guidance, Navigation \& Control, Delft University of Technology, Delft, The Netherlands, Apr. 10-12 2013, pp. 91-107.

[14] D. Izzo, N. Weiss, and T. Seidl, "Constant-Optic-Flow Lunar Landing: Optimality and Guidance," Journal of Guidance, Control, and Dynamics, vol. 34, pp. 1383-1395, 2011.

[15] D. Izzo and G. de Croon, "Landing with time-to-contact and ventral optic flow estimates," Journal of Guidance, Control, and Dynamics, vol. 35 (4), pp. 1362-1367, 2011.

[16] G. Sabiron, T. Raharijaona, L. Burlion, E. Kervendal, E. Bornschlegl, and F. Ruffier, "Sub-optimal Lunar Landing GNC using Non-gimbaled Bio-inspired Optic Flow Sensors,' IEEE Transactions on Aerospace and Electronic Systems, (in revision).

[17] T. Jean-Marius and S. Trinh, "Integrated Vision and Navigation for Planetary Exploration - Final Report," Aérospatiale Espace \& Défense Tech. Rep. RM-TN-00-18-AS/M, 1999.

[18] J. Koenderink and A. Doorn, "Facts on optic flow," Biological Cybernetics, vol. 56, pp. 247-254, 1987.

[19] A. Argyros, D. Tsakiris, and C. Groyer, "Biomimetic centering behavior [mobile robots with panoramic sensors]," Robotics Automation Magazine, vol. 11, no. 4, pp. 21 - 30, 68, Dec. 2004.

[20] P. Falugi, E. Kerrigan, and E. Van Wyk, Imperial College London Optimal Control Software User Guide (ICLOCS), Department of Electrical Engineering, Imperial College London, London, UK, 2010.

[21] H. K. Khalil and J. Grizzle, Nonlinear systems. Prentice hall Upper Saddle River, 2002, vol. 3.

[22] G. Sabiron, J. G., E. Kervendal, E. Bornschlegl, T. Raharijaona, and F. Ruffier, "Backup State Observer Based on Optic Flow Applied to Lunar Landing," in IEEE/RSJ International Conference on Intelligent Robots and Systems (IROS) (In press), 2014. 